Ark. Mat., 43 (2005), 145-165

(c) 2005 by Institut Mittag-Leffler. All rights reserved

\title{
Formulae for the distance in some quasi-Banach spaces
}

\author{
David E. Edmunds and Georgi E. Karadzhov
}

\begin{abstract}
Let $\left(A_{0}, A_{1}\right)$ be a compatible pair of quasi-Banach spaces and let $A$ be a corresponding space of real interpolation type such that $A_{0} \cap A_{1}$ is not dense in $A$. Upper and lower estimates are obtained for the distance of any element $f$ of $A$ from $A_{0} \cap A_{1}$. These lead to formulae for the distance in a large number of concrete situations, such as when $A_{0} \cap A_{1}=L^{\infty}$ and $A$ is either weak- $L^{q}$, a 'grand' Lebesgue space or an Orlicz space of exponential type.
\end{abstract}

\section{Introduction}

Let $\vec{A}=\left(A_{0}, A_{1}\right)$ be a compatible pair of quasi-Banach spaces; that is, both $A_{0}$ and $A_{1}$ are continuously embedded in some quasi-Banach space $\Sigma_{A}$. For $0<\theta<1$ and $0<p \leq \infty$ let $\vec{A}_{\theta, p}\left(=\left(A_{0}, A_{1}\right)_{\theta, p}\right)$ be the real interpolation space defined by means of the Peetre $K$-functional. It is well known that if $p<\infty$, then $A_{0} \cap A_{1}$ is dense in $\vec{A}_{\theta, p}$, while in general, $A_{0} \cap A_{1}$ is not dense in $\vec{A}_{\theta, \infty}$. The problem of quantifying this lack of density thus arises: in other words, given any $f \in \vec{A}_{\theta, \infty}$, is it possible to obtain usable upper and lower estimates for inf $\|f-g\|_{\vec{A}_{\theta, \infty}}$, where the infirmum is taken over all $g \in A_{0} \cap A_{1}$ ?

Among those who have studied the estimation of the distance of the elements of a function space from a subspace of that space are Garnett and Jones [6], who gave upper and lower estimates for the distance of elements of $\operatorname{BMO}\left(\mathbf{R}^{n}\right)$ to $L^{\infty}\left(\mathbf{R}^{n}\right)$. More recently, Carozza and Sbordone [4] did the same for other spaces close to $L^{\infty}(\Omega)$ ( $\Omega$ being an open subset of $\mathbf{R}^{n}$ ), notably weak- $L^{q}(\Omega)$, certain 'grand' Lebesgue spaces and Orlicz spaces with exponential Young functions.

In this paper we give a positive answer to the question raised above, even in settings considerably more general than those described. The abstract results obtained give rise to formulae for the distance in a wide variety of concrete situations. To explain this, let $A_{0}$ and $A_{1}$ be as above, let $f \in A_{0}+A_{1}$ and let $t \mapsto K(t, f)=K\left(t, f ; A_{0}, A_{1}\right)$ be Peetre's $K$-functional. Let $w:(0, \infty) \rightarrow \mathbf{R}$ be posi- 
tive and continuous, with $w(t) \min \{1, t\} \rightarrow 0$ as $t \rightarrow 0$ and as $t \rightarrow \infty$; and suppose that $w$ is equivalent to a non-increasing function, while $t \mapsto t w(t)$ is equivalent to a non-decreasing function on $(0, \infty)$. Corresponding to the classical real interpolation spaces defined by means of the function $t \mapsto t^{-\theta}(0<\theta<1)$, we define $\vec{A}_{w, \infty}$ to be the space of all $f \in A_{0}+A_{1}$ for which the quasi-norm

$$
\|f\|_{\vec{A}_{w, \infty}}:=\sup _{0<t<\infty} w(t) K(t, f)
$$

is finite. Endowed with this quasi-norm, $\vec{A}_{w, \infty}$ is a quasi-Banach space. Given any $f \in \vec{A}_{w, \infty}$, its distance from $A_{0} \cap A_{1}$, measured in $\vec{A}_{w, \infty}$, is

$$
\operatorname{dist}_{\vec{A}_{w, \infty}}\left(f, A_{0} \cap A_{1}\right):=\inf \left\{\|f-g\|_{\vec{A}_{w, \infty}}: g \in A_{0} \cap A_{1}\right\} .
$$

We give upper and lower estimates for this distance. For example, when $A_{0}$ and $A_{1}$ are Banach spaces with $A_{1} \subset A_{0}$, the norm of the embedding being 1 , and $w(t)=t^{-\theta}$, $0<\theta<1$, we show that

$$
\limsup _{t \rightarrow 0} t^{-\theta} K(t, f) \leq \operatorname{dist}_{\vec{A}_{\theta, \infty}}\left(f, A_{1}\right) \leq 2 \limsup _{t \rightarrow 0} t^{-\theta} K(t, f) .
$$

Corresponding results are given for the case $A_{0} \subset A_{1}$, and also for the general situation when $\left(A_{0}, A_{1}\right)$ is simply a compatible pair of quasi-Banach spaces.

With the exception of Remark 2.4, our abstract theorems, such as those just mentioned, give only equivalence formulae for the distance. However, they provide important guides for the establishment of the precise formulae for the distance in the numerous concrete situations which are considered. A special case of the results established here is that the distance of an element $f$ of weak- $L^{q}(\Omega)$ from $L^{\infty}(\Omega)$, when $\Omega$ has finite measure and $0<q<\infty$, is precisely

$$
\limsup _{t \rightarrow 0} t^{1 / q} f^{*}(t)
$$

Here $f^{*}$ is the familiar non-increasing rearrangement of $f$ (see [2], Chapter 2). This particular result, with the restriction $q>1$, was given in [4]. Sequential versions of our theorems enable us to handle similar questions related to ideals of compact operators, although we shall not do this here.

Cases of particular importance arise when the interpolation space $\vec{A}_{w, \infty}$ can be characterised as an extrapolation space. We mention here two special examples of what we prove. First, suppose that $\Omega$ has finite measure and that $a>0$. Then for all $f$ in the Zygmund space $X:=L^{\infty}(\log L)_{-a}(\Omega)$, which is actually an Orlicz space of exponential type,

$$
\operatorname{dist}_{X}\left(f, L^{\infty}(\Omega)\right)=\limsup _{\sigma \rightarrow 0} \sigma^{a}\|f\|_{L^{1 / \sigma}(\Omega)}=\limsup _{t \rightarrow 0}|\log t|^{-a} f^{*}(t) .
$$


A similar result is contained in [4]. Second, if $0<q<\infty$ and $L^{q)}(\Omega)$ is the 'grand' Lebesgue space introduced by Iwaniec and Sbordone [7], then

$$
\operatorname{dist}_{L^{q)}(\Omega)}\left(f, L^{\infty}(\Omega)\right)=\limsup _{\sigma \rightarrow 0} \sigma^{1 / q}\|f\|_{L^{q-\sigma}(\Omega)},
$$

a result proved, when $1<q<\infty$, in [4].

\section{The distance in interpolation spaces}

First we recall some definitions. Let $\vec{A}=\left(A_{0}, A_{1}\right)$ be a compatible pair of quasiBanach spaces, so that $A_{0}$ and $A_{1}$ are continuously embedded in some quasi-Banach space $\Sigma_{A}$. Let $\vec{A}_{\theta, p}(0<\theta<1,0<p \leq \infty)$ denote the real interpolation scale of Lions and Peetre, defined by the $K$-method.

If we replace $t^{-\theta}$ in this definition by a more general positive and continuous weight $w(t)$, we can define the corresponding real interpolation spaces $\vec{A}_{w, p}$ associated with the $K$-method in the familiar way using the quasi-norms

$$
\|f\|_{\vec{A}_{w, p}}=\left(\int_{0}^{\infty}[w(t) K(t, f ; \vec{A})]^{p} \frac{d t}{t}\right)^{1 / p}
$$

with the natural interpretation when $p=\infty$. Here

$$
K(t, f ; \vec{A}):=\inf _{f=f_{0}+f_{1}}\left(\left\|f_{0}\right\|_{A_{0}}+t\left\|f_{1}\right\|_{A_{1}}\right), \quad f \in A_{0}+A_{1} .
$$

We shall suppose that the weight $w$ has the following properties:

$$
\begin{aligned}
& \min \{1, t\} w(t) \rightarrow 0 \text { as } t \rightarrow 0 \text { and as } t \rightarrow \infty, \\
& w \text { is equivalent to a non-increasing function on }(0, \infty), \\
& t \mapsto t w(t) \text { is equivalent to a non-decreasing function on }(0, \infty) \text {. }
\end{aligned}
$$
$A_{0} \cap A_{1}$ :

Denote by dist $\vec{A}_{w, \infty}\left(f, A_{0} \cap A_{1}\right)$ the distance of $f$ in $\vec{A}_{w, \infty}$ to the intersection

$$
\operatorname{dist}_{\vec{A}_{w, \infty}}\left(f, A_{0} \cap A_{1}\right):=\inf _{g \in A_{0} \cap A_{\perp}}\|f-g\|_{\vec{A}_{w, \infty}} .
$$

Note that in this definition we can replace the space $A_{0} \cap A_{1}$ by any dense subset. Let

$$
\begin{aligned}
& d_{1}(f):=\limsup _{t \rightarrow 0} w(t) K(t, f), \\
& d_{2}(f):=\limsup _{t \rightarrow \infty} w(t) K(t, f)
\end{aligned}
$$


and

$$
d(f):=\max \left\{d_{1}(f), d_{2}(f)\right\}
$$

where $K(t, f):=K\left(t, f ; A_{0}, A_{1}\right):=K(t, f ; \vec{A})$.

We recall that a $p$-norm on a linear space $X$ is a map $\|\cdot\|: X \rightarrow \mathbf{R}_{+}$which satisfies the norm axioms with the triangle inequality replaced by the condition

$$
\|x+y\|^{p} \leq\|x\|^{p}+\|y\|^{p}, \quad x, y \in X
$$

Given any quasi-norm $\|\cdot\|_{1}$ on $X$, there is an equivalent $p$-norm on $X$ (see, for example, [5]): the particular value of $p$ is given by the relation $C=2^{1 / p-1}$, where $C$ is the best constant in the quasi-triangle inequality

$$
\|x+y\|_{1} \leq C\left(\|x\|_{1}+\|y\|_{1}\right)
$$

Of course, $p=1$ when $X$ is a Banach space. Henceforth the value of $p$ will be that determined in the way just explained. A quasi-Banach space equipped with the corresponding $p$-norm will be called a $p$-Banach space.

We begin with the $p$-Banach case, that is, when both $A_{0}$ and $A_{1}$ are $p$-Banach spaces. Then $f \mapsto K(t, f ; \vec{A})$ is a $p$-norm for all $t>0$. It turns out that if we measure the distance to the intersection $A_{1} \cap \vec{A}_{w, \infty}$, then the functional $d_{1}(f)$ suffices.

Note the particular case when $A_{1} \subset A_{0}$, the $p$-norm of the embedding being 1. Then $K(t, f)=\|f\|_{0}$ for $t \geq 1$ (we use the abbreviations $\|f\|_{j}=\|f\|_{A_{j}}$ for $j=0,1)$. Hence, if $w$ satisfies $(2.2)$, then the $p$-norm of $f$ in $\vec{A}_{w, \infty}$ is equivalent to $\sup _{0<t \leq 1} w(t) K(t, f)$. Thus the values of $w$ on the interval $(1, \infty)$ are not important and we can take $w(t)=0$ for $t \geq 1$ and define

$$
\|f\|_{\vec{A}_{w, \infty}}=\sup _{0<t \leq 1} w(t) K(t, f) .
$$

Analogously, if $A_{0} \subset A_{1}$, with the $p$-norm of the embedding being 1 , then $K(t, f)=$ $t\|f\|_{1}$ for $0<t<1$, and so now we define the $p$-norm in $\vec{A}_{w, \infty}$ by

$$
\|f\|_{\vec{A}_{w, \infty}}=\sup _{t \geq 1} w(t) K(t, f) .
$$

Theorem 2.1. (The $p$-Banach case) Let $w$ have the properties (2.1), (2.2) and $(2.3)$. Then for all $f \in \vec{A}_{w, \infty}$,

$$
d_{1}(f) \leq \operatorname{dist}_{\vec{A}_{w, \infty}}\left(f, A_{1} \cap \vec{A}_{w, \infty}\right) \leq \max \left\{b_{1 w},\left(1+c_{1 w}^{p}\right)^{1 / p}\right\} d_{1}(f)
$$


where

$$
b_{1 w}:=\lim _{\alpha \rightarrow 0} \sup _{\alpha \leq t<\infty} g_{\alpha}(t), \quad g_{\alpha}(t):=\frac{w(t)}{w(\alpha)}
$$

and

$$
\epsilon_{1 w}:=\lim _{\alpha \rightarrow 0} \sup _{0<t \leq \alpha} h_{\alpha}(t), \quad h_{\alpha}(t):=\frac{t w(t)}{\alpha w(\alpha)} .
$$

Proof. Since for any $g \in A_{1}$ we have

$$
K^{p}(t, f) \leq K^{p}(t, g)+K^{p}(t, f-g) \leq t^{p}\|g\|_{.2}^{p}+K^{p}(t, f-g),
$$

the left inequality of (2.8) follows. To complete the proof, we take any $\varepsilon>0$ and for any $\alpha>0$ choose $f_{\alpha} \in A_{1}$ so that

$$
\left\|f-f_{\alpha}\right\|_{0}+\alpha\left\|f_{\alpha}\right\|_{1} \leq(1+\varepsilon) K(\alpha, f) .
$$

Let

$$
I(\alpha):=\sup _{0<t \leq \alpha} w(t) K(t, f)
$$

Since

$$
\left\|f-f_{\alpha}\right\|_{\vec{A}_{w, \infty}}=\max \left\{\sup _{0<t<\alpha} w(t) K\left(t, f-f_{\alpha}\right), \sup _{\alpha<t<\infty} w(t) K\left(t, f-f_{\alpha}\right)\right\}
$$

and

$$
K\left(t, f-f_{\alpha}\right) \leq\left\|f-f_{\alpha}\right\|_{0} \leq(1+\varepsilon) K(\alpha, f),
$$

we have

$$
\sup _{\alpha<t<\infty} w(t) K\left(t, f-f_{\alpha}\right) \leq(1+\varepsilon) I(\alpha) \sup _{\alpha<t<\infty} g_{\alpha}(t) .
$$

On the other hand,

$$
\sup _{0<t<\alpha}\left[w(t) K\left(t, f-f_{\alpha}\right)\right]^{p} \leq I^{p}(\alpha)+\sup _{0<t<\alpha}\left[w(t) K\left(t, f_{\alpha}\right)\right]^{p}
$$

and

$$
\sup _{0<t<\alpha} w(t) K\left(t, f_{\alpha}\right) \leq \sup _{0<t<\alpha} t w(t)\left\|f_{\alpha}\right\|_{1} \leq(1+\varepsilon) I(\alpha) \sup _{0<t<\alpha} h_{\alpha}(t) .
$$

Hence

$$
\sup _{0<t<\alpha} w(t) K\left(t, f-f_{\alpha}\right) \leq I(\alpha)\left(1+(1+\varepsilon)^{p} \sup _{0<t<\alpha} h_{\alpha}^{p}(t)\right)^{1 / p} .
$$

Thus $f-f_{\alpha} \in \vec{A}_{w, \infty}$. In particular, $f_{\alpha} \in \vec{A}_{w, \infty}$ and therefore $\operatorname{dist}_{\vec{A}_{w, \infty}}\left(f, A_{1} \cap \vec{A}_{w, \infty}\right)$ is bounded from above by

$$
I(\alpha) \max \left\{(1+\varepsilon) \sup _{\alpha<t<\infty} g_{\alpha}(t),\left(1+(1+\varepsilon)^{p} \sup _{0<t<\alpha} h_{\alpha}^{p}(t)\right)^{1 / p}\right\} .
$$

Now take the limit as $\alpha \rightarrow 0$ and then as $\varepsilon \rightarrow 0$.

Analogously, if we measure the distance to the intersection $A_{0} \cap \vec{A}_{w, \infty}$, then the functional $d_{2}(f)$ suffices. 
Theorem 2.2. (The $p$-Banach case) Let $w$ have the properties (2.1), (2.2) and (2.3). Then for all $f \in \vec{A}_{w, \infty}$,

$$
d_{2}(f) \leq \operatorname{dist}_{\vec{A}_{w, \infty}}\left(f, A_{0} \cap \vec{A}_{w, \infty}\right) \leq \max \left\{b_{2 w},\left(1+c_{2 w}^{p}\right)^{1 / p}\right\} d_{2}(f)
$$

where

$$
b_{2 w}:=\lim _{\beta \rightarrow \infty} \sup _{0 \leq t \leq \beta} h_{\beta}(t)
$$

and

$$
c_{2 w}:=\lim _{\beta \rightarrow \infty} \sup _{t \geq \beta} g_{\beta}(t)
$$

Theorem 2.3. (The quasi-Banach case) Let $w$ have properties (2.1), (2.2) and (2.3). Then for all $f \in \vec{A}_{w, \infty}$,

$$
\frac{1}{c_{A}} d(f) \leq \operatorname{dist}_{\vec{A}_{w, \infty}}\left(f, A_{0} \cap A_{1}\right) \leq c d(f)
$$

where $c_{A}$ is the best constant in the quasi-triangle inequality

$$
\begin{gathered}
K(t, g-h) \leq c_{A}[K(t, g)+K(t, h)], \quad g, h \in A_{0}+A_{1}, \\
c:=c_{A} \max \left\{c_{w}, 1+\max \left\{c_{1 w}, c_{2 w}\right\}\right\}, \quad c_{w}:=\lim _{\substack{\alpha \rightarrow 0 \\
\beta \rightarrow \infty}} \sup _{\alpha \leq t \leq \beta} h_{\alpha, \beta}(t)
\end{gathered}
$$

and

$$
h_{\alpha, \beta}(t):=w(t)\left(\frac{1}{w(\alpha)}+\frac{t}{\beta w(\beta)}\right) \quad \text { for } 0<\alpha \leq t \leq \beta, \alpha<1<\beta
$$

Proof. Let $f \in \vec{A}_{w, \infty}$. Since for any $g \in A_{0} \cap A_{1}$ with $\left(A_{0} \cap A_{1}\right)$-norm $\|g\|$ we have

$$
\frac{1}{c_{A}} K(t, f) \leq \min \{1, t\}\|g\|+K(t, f-g),
$$

the left inequality of (2.14) follows. To prove the remainder we take any $\varepsilon>0$ and choose a representation $f=f_{0}(s)+f_{1}(s)\left(f_{0}(s) \in A_{0}, f_{1}(s) \in A_{1}\right)$ such that

$$
\left\|f_{0}(s)\right\|_{0}+s\left\|f_{1}(s)\right\|_{1} \leq(1+\varepsilon) K(s, f)
$$


for all $s>0$. Here $\|h\|_{j}:=\|h\|_{A_{j}}$ for $j=0,1$. For all integers $\nu$ put $f_{j, \nu}=f_{j}\left(2^{\nu}\right)$ and $g_{\nu}=f_{0, \nu}-f_{0, \nu-1}$; then for any $M>M_{0}$ and $N>N_{0}$, say,

$$
g:=\sum_{\nu=-N}^{M} g_{\nu}=f_{0, M}-f_{0,-N-1}=f_{1,-N-1}-f_{1, M} \in A_{0} \cap A_{1},
$$

and

$$
f-g=f_{0,-N-1}+f_{1, M} .
$$

Let $\alpha:=2^{-N-1}, \beta:=2^{M}$ and let

$$
I(\alpha, \beta):=\max \left\{\sup _{0<t \leq \alpha} w(t) K(t, f), \sup _{t \geq \beta} w(t) K(t, f)\right\} .
$$

Then

$$
K(t, f-g) \leq\left\|f_{0,-N-1}\right\|_{0}+t\left\|f_{1, M}\right\|_{1} \leq(1+\varepsilon)\left(K(\alpha, f)+\frac{t}{\beta} K(\beta, f)\right)
$$

and so

$$
w(t) K(t, f-g) \leq(1+\varepsilon) I(\alpha, \beta) h_{\alpha, \beta}(t) .
$$

Hence

$$
\sup _{\alpha \leq t \leq \beta} w(t) K(t, f-g) \leq(1+\varepsilon) I(\alpha, \beta) \sup _{\alpha \leq t \leq \beta} h_{\alpha, \beta}(t) .
$$

On the other hand, for $t>\beta$ or $t<\alpha$ we have

$$
\frac{1}{c_{A}} w(t) K(t, f-g) \leq I(\alpha, \beta)+w(t) K(t, g) .
$$

Now we estimate $\|g\|$. We have

$$
\|g\|_{0} \leq\left\|f_{0, M}\right\|_{0}+\left\|f_{0,-N-1}\right\|_{0} \leq(1+\varepsilon)[K(\beta, f)+K(\alpha, f)]
$$

and

$$
\|g\|_{1} \leq\left\|f_{1, M}\right\|_{1}+\left\|f_{1,-N-1}\right\|_{1} \leq(1+\varepsilon)\left(\frac{K(\beta, f)}{\beta}+\frac{K(\alpha, f)}{\alpha}\right) .
$$

Hence if $t>\beta$ we obtain

$$
w(t) K(t, g) \leq w(t)\|g\|_{0} \leq(1+\varepsilon) I(\alpha, \beta) h_{\alpha, \beta}(\beta) \frac{w(t)}{w(\beta)},
$$


and for $t<\alpha$ we have

$$
w(t) K(t, g) \leq t w(t)\|g\|_{1} \leq(1+\varepsilon) I(\alpha, \beta) h_{\alpha, \beta}(\alpha) \frac{t w(t)}{\alpha w(\alpha)} .
$$

This and estimates (2.16) and (2.17) show that $c_{A}^{-1}\|f-g\|_{\vec{A}_{w, \infty}}[(1+\varepsilon) I(\alpha, \beta)]^{-1}$ is bounded above by

$$
\max \left\{\sup _{\alpha \leq t \leq \beta} h_{\alpha, \beta}(t), 1+\max \left\{\sup _{0<t \leq \alpha} h_{\alpha}(t) h_{\alpha, \beta}(\alpha), \sup _{t \geq \beta} g_{\beta}(t) h_{\alpha, \beta}(\beta)\right\}\right\},
$$

where $g_{\alpha}$ and $h_{\alpha}$ are defined by (2.9) and (2.10). The right inequality of (2.14) now follows and the proof is complete.

Remark 2.4. The weights $w(t)=1$ and $w(t)=1 / t$ do not satisfy condition (2.1). However, we can prove directly that

$$
\operatorname{dist}_{A_{0}}\left(f, A_{0} \cap A_{1}\right)=\limsup _{t \rightarrow 0} K\left(t, f ; A_{0}, A_{1}\right)
$$

and

$$
\operatorname{dist}_{A_{1}}\left(f, A_{0} \cap A_{1}\right)=\limsup _{t \rightarrow \infty} \frac{\mathrm{K}\left(t, f ; A_{0}, A_{1}\right)}{t} .
$$

Proof. If $f \in A_{0}$ then

$$
K\left(t, f ; A_{0}, A_{1}\right)=\inf _{f_{1} \in A_{0} \cap A_{1}}\left(\left\|f-f_{1}\right\|_{0}+t\left\|f_{1}\right\|_{1}\right) .
$$

Hence

$$
K\left(t, f ; A_{0}, A_{1}\right) \geq \inf _{f_{1} \in A_{0} \cap A_{1}}\left\|f-f_{1}\right\|_{0},
$$

which proves half of (2.18). On the other hand,

$$
K\left(t, f ; A_{0}, A_{1}\right) \leq\left\|f-f_{1}\right\|_{0}+t\left\|f_{1}\right\|_{1}, \quad f_{1} \in A_{0} \cap A_{1},
$$

and so

$$
\limsup _{t \rightarrow 0} K\left(t, f ; A_{0}, A_{1}\right) \leq\left\|f-f_{1}\right\|_{0}, \quad f_{1} \in A_{0} \cap A_{1},
$$

which completes the proof of (2.18). The proof of (2.19) is similar.

As illustrations of these results, consider the pair $\left(L^{1}(R), L^{\infty}(R)\right)$, where $R$ is an arbitrary $\sigma$-finite measure space. Then using Remark 2.4 and the fact that

$$
K\left(t, f ; L^{1}(R), L^{\infty}(R)\right)=\int_{0}^{t} f^{*}(s) d s
$$


(see [11], p. 133), we have the following formula for the distance of $f \in L^{\infty}(R)$ to the intersection $L^{1}(R) \cap L^{\infty}(R)$ :

$$
\operatorname{dist}_{L^{\infty}(R)}\left(f, L^{1}(R) \cap L^{\infty}(R)\right)=\limsup _{t \rightarrow \infty} \frac{1}{t} \int_{0}^{t} f^{*}(s) d s=f^{*}(\infty) .
$$

In a similar way we see that

$$
\operatorname{dist}_{l \infty}\left(f, l^{1}\right)=\limsup _{n \rightarrow \infty} \frac{1}{n} \sum_{k=1}^{n} f^{*}(k)=f^{*}(\infty) .
$$

In particular, since $l^{1}$ is dense in the subspace $c_{0} \subset l^{\infty}$, it follows that

$$
\operatorname{dist}_{l \infty}\left(f, c_{0}\right)=f^{*}(\infty)
$$

Now let $A$ be a quasi-Banach space which is intermediate for $\left(A_{0}, A_{1}\right)$; that is, $A_{0} \cap A_{1} \subset A \subset A_{0}+A_{1}$. Denote by $A^{\circ}$ the closure of $A_{0} \cap A_{1}$ in $A$. Using Theorem 2.3 we can characterise the space $\left(\vec{A}_{w, \infty}\right)^{\circ}$ as follows:

Theorem 2.5. Let $w$ satisfy (2.1), (2.2) and (2.3). Then

$$
\left(\vec{A}_{w, \infty}\right)^{o}=\left\{f \in \vec{A}_{w, \infty}: d(f)=0\right\} .
$$

\section{Examples of concrete spaces}

Here we consider some particular spaces in which a direct approach gives precise formulae for the distance (cf. [4]).

Let $b$ be a positive continuous function on the interval $[1, \infty)$. We say that $b$ is slowly varying on $[1, \infty)$ (in the sense of Karamata) if for all $\varepsilon>0$, the function $t \mapsto t^{\varepsilon} b(t)$ is equivalent to a non-decreasing function and $t \mapsto t^{-\varepsilon} b(t)$ is equivalent to a non-increasing function. By symmetry, we say that a positive continuous function $b$ on the interval $(0,1]$ is slowly varying on $(0,1]$ if the function $t \rightarrow b(1 / t)$ is slowly varying on $[1, \infty)$. Finally, a positive continuous function on $(0, \infty)$ is said to be slowly varying on $(0, \infty)$ if it is slowly varying on both $(0,1]$ and $[1, \infty)$.

Let $(\Omega, \mu)$ be a $\sigma$-finite measure space and let $b$ be slowly varying on $(0, \infty)$. Then if $0<q \leq \infty$ and $0<r \leq \infty$, the Lorentz-Karamata space $L_{b}^{q, r}(\Omega)$ (see [10]) is defined to be the set of all functions $f$ on $\Omega$ for which the quasi-norm

$$
\|f\|_{L_{b}^{q, r}(\Omega)}:=\left(\int_{0}^{\mu(\Omega)}\left[t^{1 / q} b(t) f^{*}(t)\right]^{r} \frac{d t}{t}\right)^{1 / r}
$$


is finite. When $r=\infty$ this is to be interpreted as

$$
\|f\|_{L_{b}^{q, \infty}(\Omega)}:=\sup _{0<t<\mu(\Omega)} t^{1 / q} b(t) f^{*}(t) .
$$

If $q=r$ we simply write $L_{b}^{q}(\Omega)$ instead of $L_{b}^{q, r}(\Omega)$, and if no ambiguity is possible we write $L_{b}^{q, r}$ for $L_{b}^{q, r}(\Omega)$.

We also need an equivalent definition in terms of the corresponding $p$-norm, where $0<p \leq 1$ and $p<q, p \leq r$. Since the distance depends on the particular quasinorm or $p$-norm being employed, we use a different notation, $L_{b,(p)}^{q, r}(\Omega)$, for the Lorentz-Karamata space when equipped with the $p$-norm

$$
\|f\|_{L_{b,(p)}^{q, r}(\Omega)}:=\left(\int_{0}^{\mu(\Omega)}\left[t^{1 / q-1 / p} b(t)\left(\int_{0}^{t} f^{*}(s)^{p} d s\right)^{1 / p}\right]^{r} \frac{d t}{t}\right)^{1 / r} .
$$

When $p=1$ this simply means that $f^{* *}(t):=t^{-1} \int_{0}^{t} f^{*}(\sigma) d \sigma$ is being used instead of $f^{*}(t)$.

Note two particular cases. If $b=1$, then we obtain the Lorentz spaces $L^{q, r}$ and $L_{(p)}^{q, r}$, while the Lorentz-Zygmund space $L^{q, r}(\log L)^{a}$ (see [1]) results from the choice $b(t)=(1+|\log t|)^{a}$.

Now suppose that $w(t):=t^{1 / q-1 / p} b(t)$ and $w\left(t^{p}\right)$ satisfy $(2.1)$, where $p$ is determined as explained above for $L_{b,(p)}^{q, \infty}(\Omega)$. Since the space $L_{b,(p)}^{q, \infty}(\Omega)$ is just the interpolation space $\left(L^{p}(\Omega), L^{\infty}(\Omega)\right)_{w\left(t^{p}\right), \infty}$, we can apply Theorem 2.1 in the case of a finite measure space and conclude that

$$
\operatorname{dist}_{L_{b,(p)}^{q, \infty}(\Omega)}\left(f, L^{\infty}(\Omega)\right) \approx d_{1,(p)}(f),
$$

where

$$
d_{1,(p)}(f):=\limsup _{t \rightarrow 0} t^{1 / q-1 / p} b(t)\left(\int_{0}^{t} f^{*}(s)^{p} d s\right)^{1 / p} .
$$

Here we use the fact (see $[11]$, p. 135) that

Moreover,

$$
K(t, f) \approx\left(\int_{0}^{t^{p}} f^{*}(s)^{p} d s\right)^{1 / p}
$$

$$
\operatorname{dist}_{L_{b}^{q, \infty}(\Omega)}\left(f, L^{\infty}(\Omega)\right) \approx d_{1}(f),
$$

where

$$
d_{1}(f):=\limsup _{t \rightarrow 0} t^{1 / q} b(t) f^{*}(t) .
$$

When the measure space is merely $\sigma$-finite, we can apply Theorem 2.3 to give

$$
\operatorname{dist}_{L_{b}^{q, \infty}(\Omega)}\left(f, L^{p}(\Omega) \cap L^{\infty}(\Omega)\right) \approx \limsup _{t \rightarrow 0} t^{1 / q} b(t) f^{*}(t)+\limsup _{t \rightarrow \infty} t^{1 / q} b(t) f^{*}(t) .
$$

We shall now prove that in (3.2) we have equality. Moreover, $\sigma$-finiteness of the measure space is enough when we estimate the distance to $L^{\infty}(\Omega) \cap L_{b,(p)}^{q, \infty}(\Omega)$. 
Theorem 3.1. Suppose that $b$ is slowly varying on $(0, \infty)$. Let $q \in(0, \infty]$ and let $p \in(0,1], p<q$, be chosen appropriately for $L_{b}^{q, \infty}(\Omega)$ as explained above. Assume further that $t^{1 / q} b(t) \rightarrow 0$ as $t \rightarrow 0$. Then for all $f \in X:=L_{b,(p)}^{q, \infty}(\Omega)$,

$$
\operatorname{dist}_{X}\left(f, L^{\infty}(\Omega) \cap X\right)=d_{1,(p)}(f) .
$$

Proof. The estimate of $d_{1,(p)}(f)$ from above is similar to that given in the proof of Theorem 2.1, using the fact that the functional

$$
K(t, f):=\left(\int_{0}^{t} f^{*}(s)^{p} d s\right)^{1 / p}
$$

is a $p$-norm. Namely,

$$
K^{p}(t, f) \leq K^{p}(t, g)+K^{p}(t, f-g)
$$

for any $g \in L^{\infty}(\Omega)$ with quasi-norm $\|g\|$, and hence

$$
w^{p}(t) K^{p}(t, f) \leq t w^{p}(t)\|g\|^{p}+\|f-g\|_{X}^{p},
$$

where $w(t):=t^{1 / q-1 / p} b(t)$. Thus $d_{1,(p)}(f) \leq\|f-g\|_{X}$ and the required estimate follows.

It remains to estimate $d_{1,(p)}(f)$ from below. We follow the proof of Theorem 2.1, but now we have a better choice for the approximating functions $f_{k}$. Thus for $f \in X$ we take any large $k$ and define

$$
I(k)=\sup _{0<t \leq \mu_{f}(k)} w(t) K(t, f),
$$

where $\mu_{f}, \mu_{f}(\lambda):=\mu\{x \in \Omega:|f(x)|>\lambda\}$, is the distribution function of $f$. Note that (since $f \in L^{p}(\Omega)+L^{\infty}(\Omega)$ )

$$
\mu_{f}(k) \rightarrow 0 \quad \text { as } k \rightarrow \infty
$$

We define $f_{k}$ by

$$
f_{k}(x)= \begin{cases}f(x), & |f(x)| \leq k, \\ 0, & \text { otherwise. }\end{cases}
$$

Hence $f-f_{k}=f$ on the set $\Omega_{k}=\{x \in \Omega:|f(x)|>k\}$, and we obtain the formulae

$$
\begin{array}{ll}
\left(f-f_{k}\right)^{*}(s)=f^{*}(s), & \text { if } s<\mu_{f}(k), \\
\left(f-f_{k}\right)^{*}(s)=0, & \text { if } s \geq \mu_{f}(k) .
\end{array}
$$


In particular,

$$
K\left(t, f-f_{k}\right)= \begin{cases}K(t, f), & \text { if } t \leq \mu_{f}(k), \\ K\left(\mu_{f}(k), f\right), & \text { if } t>\mu_{f}(k) .\end{cases}
$$

For shortness, put $J(t, k)=w(t) K\left(t, f-f_{k}\right)$. Then

$$
\left\|f-f_{k}\right\|_{X}=\max \left\{\sup _{0<t<\mu_{f}(k)} J(t, k), \sup _{t>\mu_{f}(k)} J(t, k)\right\},
$$

and so, using the monotonicity of $w,(3.8)$ and the estimates above,

$$
\left\|f-f_{k}\right\|_{X} \leq \max \left\{\sup _{0<t<\mu_{f}(k)} w(t) K(t, f), \sup _{t>\mu_{f}(k)} w\left(\mu_{f}(k)\right) K\left(\mu_{f}(k), f\right)\right\} \leq I(k) .
$$

Application of (3.9) now gives the desired estimate

$$
\operatorname{dist}_{X}\left(f, L^{\infty}(\Omega) \cap X\right) \leq d_{1,(p)}(f) .
$$

We observe that a similar result is established in [4] for the Banach case $q>1$, with $b=1$.

It turns out that we can also prove equality in formula (3.4) if, in addition, $t^{-N} b(t)$ is non-increasing for some $N>1$. To cover this case, we notice that the quasi-norm, although not a $p$-norm any more, has a special property that will suffice, namely that

$$
v(t) f^{*}(t) \leq\|f-g\|_{L_{b}^{q, \infty}+v(t)\|g\|_{L^{\infty}}}
$$

where $v(t):=t^{1 / q} b(t)$ and it is assumed that $t^{-N} b(t)$ is non-increasing for some $N>1$.

Theorem 3.2. Let $b$ be slowly varying on $(0, \infty)$ and suppose that $v(t):=$ $t^{1 / q} b(t) \rightarrow 0$ as $t \rightarrow 0$; assume additionally that for some $N>1, t^{-N} b(t)$ is nonincreasing. Then for all $f \in L_{b}^{q, \infty}$,

$$
\text { dist }_{L_{b}^{q, \infty}}\left(f, L^{\infty} \cap L_{b}^{q, \infty}\right)=\limsup _{t \rightarrow 0} v(t) f^{*}(t) .
$$

Proof. For any small $\varepsilon>0$ and $g \in L^{\infty}$ we have

$$
v(t) f^{*}(t) \leq v(t)(f-g)^{*}((1-\varepsilon) t)+v(t) g^{*}(\varepsilon t),
$$

from which, using the monotonicity property of $b$, we obtain

$$
\begin{aligned}
v(t) f^{*}(t) & \leq(1-\varepsilon)^{-N-1 / q} v((1-\varepsilon) t)(f-g)^{*}((1-\varepsilon) t)+v(t) g^{*}(\varepsilon t) \\
& \leq(1-\varepsilon)^{-N-1 / q}\|f-g\|_{L_{b}^{q, \infty}+v(t)\|g\|_{L^{\infty}} .}
\end{aligned}
$$


Taking the limit as $t \rightarrow 0$, we see that

$$
d_{1}(f) \leq(1-\varepsilon)^{-N-1 / q}\|f-g\|_{L_{b}^{q, \infty}}
$$

which gives the estimate (3.13) from below. The reverse estimate is proved using the same approach as in the proof of Theorem 3.1. We let $f \in L_{b}^{q, \infty}$, and observe that $f \in L^{p}+L^{\infty}$, where $p<q$, so that $\mu_{f}(k) \rightarrow 0$ as $k \rightarrow \infty$. Again we choose the approximating functions $f_{k}$ to be

$$
f_{k}(x)= \begin{cases}f(x), & |f(x)| \leq k \\ 0, & \text { otherwise }\end{cases}
$$

Using (3.10) and (3.11) we have

$$
\left\|f-f_{k}\right\|_{L_{b}^{q}, \infty} \leq \sup _{0<t \leq \mu_{f}(k)} v(t) f^{*}(t)
$$

Hence

$$
\operatorname{dist}_{L_{b}^{q, \infty}}\left(f, L^{\infty} \cap L_{b}^{q, \infty}\right) \leq \sup _{0<t \leq \mu_{f}(k)} v(t) f^{*}(t),
$$

and it remains to take the limit as $k \rightarrow \infty$.

\section{The distance in extrapolation spaces}

First we recall some definitions from [8] and [9]. Let $\left\{A_{\sigma}\right\}, 0<\sigma<\varepsilon \leq 1$, be a scale of compatible quasi-Banach spaces. This means that there exist quasiBanach spaces $\Delta_{A}$ and $\Sigma_{A}$ such that $\Delta_{A} \subset A_{\sigma} \subset \Sigma_{A}$ for all $\sigma \in(0, \varepsilon)$, the quasinorms of the embeddings being uniformly bounded. Let $M:[0, \varepsilon] \rightarrow \mathbf{R}$ be positive and continuous. Then the $\Delta$-extrapolation space $\Delta_{0, \varepsilon}^{(\infty)}\left(M(\sigma) A_{\sigma}\right)$ consists of all elements $f \in \bigcap_{0<\sigma<\varepsilon} A_{\sigma}$ such that

$$
\|f\|_{\Delta_{0, \varepsilon}^{(\infty)}\left(M(\sigma) A_{\sigma}\right)}:=\sup _{0<\sigma<\varepsilon} M(\sigma)\|f\|_{A_{\sigma}}
$$

is finite.

Suppose that $\left\{w_{\sigma}\right\}, 0<\sigma<\varepsilon$, is a family of positive continuous functions on the interval $(0, \infty)$ such that

$$
\sup _{0<\sigma<\varepsilon} \sup _{0<t<\infty} \min \{1, t\} w_{\sigma}(t)<\infty \text { and } \inf _{0<\sigma<\varepsilon} w_{\sigma}(1)>0
$$


Then the interpolation space $\vec{A}_{w_{\sigma}, \infty}$ is well defined and $A_{0} \cap A_{1} \subset \vec{A}_{w_{\sigma}, \infty} \subset A_{0}+A_{1}$, uniformly with respect to $\sigma \in(0, \varepsilon)$. Hence we can define the extrapolation space $\Delta_{0, \varepsilon}^{(\infty)}\left(M(\sigma) \vec{A}_{w_{\sigma}, \infty}\right)$. It is clear that

$$
\Delta_{0, \varepsilon}^{(\infty)}\left(M(\sigma) \vec{A}_{w_{\sigma}, \infty}\right)=\vec{A}_{w, \infty}
$$

where

$$
w(t)=\sup _{0<\sigma<\varepsilon} M(\sigma) w_{\sigma}(t)
$$

Suppose, moreover, that

$$
\limsup _{\sigma \rightarrow 0} M(\sigma) \sup _{0<t<\infty} \min \{1, t\} w_{\sigma}(t)=0 .
$$

From the definition of $w(t)$ there follows the existence of some $\sigma(t)$ such that

$$
M(\sigma(t)) w_{\sigma(t)} \approx w(t), \quad 0<t<\infty
$$

We shall require that

$$
\sigma(t) \rightarrow 0 \quad \text { as } t \rightarrow 0 \text { and as } t \rightarrow \infty \text {. }
$$

We also introduce the function

$$
D(f):=\limsup _{\sigma \rightarrow 0} M(\sigma)\|f\|_{\vec{A}_{w_{\sigma}, \infty}} .
$$

Theorem 4.1. Suppose the weight $w_{\sigma}$ satisfies (4.1), (4.3) and (4.5). If $w$, defined by (4.2), satisfies (2.1), (2.2) and (2.3), then

$$
\operatorname{dist}_{\vec{A}_{w, \infty}}\left(f, A_{0} \cap A_{1}\right) \approx D(f) .
$$

Proof. Since for any $g \in A_{0} \cap A_{1}$ with quasi-norm $\|g\|$ we have

$$
c K(t, f) \leq \min \{1, t\}\|g\|+K(t, f-g),
$$

the estimate of the distance from below in (4.7) follows from (4.3). To prove the reverse estimate we notice that

$$
c w(t) K(t, f) \leq M(\sigma(t)) w_{\sigma(t)}(t) K(t, f) \leq M(\sigma(t))\|f\|_{\vec{A}_{w_{\sigma(t)}, \infty}} .
$$


Hence taking the limit as $t \rightarrow 0$ or $t \rightarrow \infty$ and using (4.5) together with Theorem 2.3, we derive $c d(f) \leq D(f)$, as desired.

In some particular cases we can give a direct proof of this result in a sharper form, with precise formulae.

We start with what we shall call the generalised grand Lebesgue-Karamata spaces. We define these to be the spaces

$$
L_{b}^{q)}(\Omega):=\Delta_{0, \varepsilon}^{(\infty)}\left(M(\sigma) L^{q-\sigma}(\Omega)\right)
$$

with quasi-norm

$$
\|f\|_{L_{b}^{q)}(\Omega)}:=\sup _{0<\sigma<\varepsilon} M(\sigma)\|f\|_{L^{q-\sigma}(\Omega)}
$$

where $M$ is a positive continuous function on $(0, \varepsilon), 0<q<\infty, L^{r}(\Omega)$ is the classical Lebesgue space on a finite measure space $(\Omega, \mu)$, with $\mu(\Omega)=1$ for simplicity, and

$$
b(t)=b_{\varepsilon}(t):=\sup _{0<\sigma<\varepsilon} M(\sigma) t^{\sigma} .
$$

The function $b$ is increasing. To ensure that $b$ is slowly varying on $(0,1)$, we require a little more of the weight $M$. We say that $M$ is tempered in the sense of [8] if

$$
M(\sigma) \approx M\left(\frac{1}{2} \sigma\right), \quad 0<\sigma<\varepsilon
$$

When $M$ has this property, $b$ is slowly varying on $(0,1)$. Indeed, $b_{\varepsilon}(t) \approx b_{\varepsilon / 2}(t)$, $0<t<1$, for

$$
b_{\varepsilon}(t) \approx \sup _{0<\sigma<\varepsilon} M\left(\frac{1}{2} \sigma\right) t^{\sigma}=\sup _{0<\sigma<\varepsilon / 2} M(\sigma) t^{2 \sigma} \leq \sup _{0<\sigma<\varepsilon / 2} M(\sigma) t^{\sigma}=b_{\varepsilon / 2}(t) \leq b_{\varepsilon}(t) .
$$

Let $\alpha>0$ be arbitrary and choose an integer $k$ so that $2^{-k} \varepsilon<\alpha$. Then

$$
t^{-\alpha} b_{\varepsilon}(t) \approx c_{\varepsilon, \alpha}(t):=\sup _{0<\sigma<\varepsilon / 2} M(\sigma) t^{\sigma-\alpha},
$$

and this function is decreasing.

When $q=\infty$, we have $L_{b}^{\infty}(\Omega):=\Delta_{0, \varepsilon}^{(\infty)}\left(M(\sigma) L^{1 / \sigma, \infty}(\Omega)\right)$, with quasi-norm

$$
\|f\|_{L_{b}^{\infty}(\Omega)}:=\sup _{0<\sigma<\varepsilon} M(\sigma)\|f\|_{L^{1 / \sigma, \infty}(\Omega)} \approx \sup _{0<t<1} b(t) f^{*}(t) .
$$

Now we define

$$
D_{q}(f)=\limsup _{\sigma \rightarrow 0} M(\sigma)\|f\|_{L^{q-\sigma}(\Omega)}, \quad D_{\infty}(f)=\limsup _{\sigma \rightarrow 0} M(\sigma)\|f\|_{L^{1 / \sigma, \infty}(\Omega)}
$$


The formula for $D_{\infty}(f)$ can be simplified if, in addition, we assume that $M(\sigma) \leq$ $c(\alpha) M(\alpha \sigma)$ for all $\alpha \approx 1$, where $\limsup _{\alpha \rightarrow 1} c(\alpha) \leq 1$. Then

$$
D_{\infty}(f)=\limsup _{\sigma \rightarrow 0} M(\sigma)\|f\|_{L^{1 / \sigma}(\Omega)} .
$$

Indeed, we have only to prove the estimate from below for $D_{\infty}(f)$. For $0<\alpha<1$ we write

$$
\int_{0}^{1} f^{*}(t)^{\alpha / \sigma} d t \leq \sup _{0<t<1} \frac{t^{\alpha} f^{*}(t)^{\alpha / \sigma}}{1-\alpha}
$$

whence

$$
M(\sigma / \alpha)\|f\|_{L^{\alpha / \sigma}(\Omega)} \leq c(\alpha)(1-\alpha)^{-\sigma / \alpha} M(\sigma)\|f\|_{L^{1 / \sigma, \infty}(\Omega)} .
$$

Letting first $\sigma \rightarrow 0$ and then $\alpha \rightarrow 1$ we obtain the desired estimate.

Theorem 4.2. Let the weight $M$ be tempered and suppose that $M(\sigma) \rightarrow 0$ as $\sigma \rightarrow 0$; let $b$ be defined by (4.8). Then for all $f \in L_{b}^{q)}(\Omega)$,

$$
\operatorname{dist}_{L_{b}^{q)}(\Omega)}\left(f, L^{\infty}(\Omega)\right)=D_{q}(f), \quad 0<q<\infty,
$$

and

$$
\operatorname{dist}_{L_{b}^{\infty}(\Omega)}\left(f, L^{\infty}(\Omega)\right)=D_{\infty}(f)
$$

Proof. Let $0<q<\infty$; the case $q=\infty$ is analogous. Since the spaces $L^{q-\sigma}(\Omega)$ $(0<\sigma<\varepsilon<q)$ are $\min \{1, q-\varepsilon\}$-normed, we have the estimate of $D_{q}(f)$ from above. Now we consider approximating functions $f_{k}$ defined as in the proof of Theorem 3.2. For any $\delta \in(0, \varepsilon)$ we have $f \in L^{q-\delta}(\Omega)$ and $\operatorname{dist}_{L^{\infty}(\Omega)}\left(f, \Delta_{0, \varepsilon}^{(\infty)}\left(M(\sigma) L^{q-\sigma}(\Omega)\right)\right)$ is bounded above by

$$
\max \left\{\sup _{0<\sigma<\delta} M(\sigma)\|f\|_{L^{q-\sigma}(\Omega)}, \sup _{\delta<\sigma<\varepsilon} M(\sigma)\left(\int_{|f(x)|>k}|f(x)|^{q-\delta} d x\right)^{1 /(q-\delta)}\right\} .
$$

The required estimate follows by letting first $k \rightarrow \infty$ and then $\delta \rightarrow 0$.

Example 1. (The grand Lebesgue spaces, cf. [7]) By definition, these are the spaces

$$
L^{q)}(\Omega):=\Delta_{0, \varepsilon}^{(\infty)}\left(\sigma^{1 / q} L^{q-\sigma}(\Omega)\right) .
$$

Thus from Theorem 4.2 we have

$$
\operatorname{dist}_{L^{q)}(\Omega)}\left(f, L^{\infty}(\Omega)\right)=\limsup _{\sigma \rightarrow 0} \sigma^{1 / q}\|f\|_{L^{q-\sigma}(\Omega)} .
$$

Note that in the Banach case $q>1$, this formula is proved in [4]. 
Example 2. (The Zygmund space of exponential type, $X:=L^{\infty}(\log L)_{-a}(\Omega)$, $a>0)$ This is the space $L_{b}^{\infty}(\Omega)$ with $b(t)=(1+|\log t|)^{-a}$. Then (4.13) and Theorem 3.2 give

$$
\operatorname{dist}_{X}\left(f, L^{\infty}(\Omega)\right)=\limsup _{\sigma \rightarrow 0} \sigma^{a}\|f\|_{L^{1 / \sigma}(\Omega)}=\limsup _{t \rightarrow 0}|\log t|^{-a} f^{*}(t)
$$

In fact, a similar result can be proved in the general case, characterising $L_{b}^{q)}(\Omega)$ as an interpolation space:

$$
L_{b}^{q)}(\Omega)=\left(L^{p}(\Omega), L^{q}(\Omega)\right)_{w, \infty}
$$

where $w(t)=t^{-1} b(t), 0<p<q<\infty$ and $b$ is defined by (4.8), $M$ being tempered. To do this, we first observe that in the definition of the spaces $L_{b}^{q)}(\Omega)$ we can replace $L^{q-\sigma}(\Omega)$ by the Lorentz space $L^{q-\sigma, q}(\Omega)$, in view of the monotonicity of this scale and the fact that $M$ is tempered. Thus

$$
\Delta_{0, \varepsilon}^{(\infty)}\left(M(\sigma) L^{q-\sigma}(\Omega)\right)=\Delta_{0, \varepsilon}^{(\infty)}\left(M(\sigma) L^{q-\sigma, q}(\Omega)\right)
$$

We need the formula

$$
(1-\theta)^{1 / q}\left(L^{p}(\Omega), L^{q}(\Omega)\right)_{\theta, q}=L^{q_{\theta}, q}(\Omega), \quad \frac{1}{q_{\theta}}=\frac{1-\theta}{p}+\frac{\theta}{q}, 0<\theta_{0}<\theta<1,
$$

in the sense of equivalent quasi-norms, the equivalence constants being independent of $\theta$. To see this we write

$$
L^{q}(\Omega)=\left(L^{p}(\Omega), L^{\infty}(\Omega)\right)_{\theta, q}, \quad \frac{1}{q}=\frac{1-\eta}{p},
$$

and use the Holmstedt formula [3]

$$
\begin{aligned}
K\left(t, f ; L^{p}(\Omega), L^{q}(\Omega)\right) & \approx t^{q} \int_{t^{1 / \eta}}^{\infty} u^{-\eta q} K^{q}(u, f) \frac{d u}{u} \\
K(u, f) & :=K\left(u, f ; L^{p}(\Omega), L^{\infty}(\Omega)\right) .
\end{aligned}
$$

Then straightforward calculation shows that

$$
\|f\|_{\left(L^{p}(\Omega), L^{q}(\Omega)\right)_{\theta_{i q}}}=[\eta(1-\theta) q]^{-1 / q}\|f\|_{\left(L^{p}(\Omega), L^{\infty}(\Omega)\right)_{\eta \theta, q}} .
$$

Thus (4.18) follows. Now we can continue the formula (4.17) as follows:

$$
\Delta_{0, \varepsilon}^{(\infty)}\left(M(\sigma) L^{q-\sigma, q}(\Omega)\right)=\Delta_{0, \varepsilon}^{(\infty)}\left(M(\sigma) \sigma^{1 / q}\left(L^{p}(\Omega), L^{q}(\Omega)\right)_{1-\alpha \sigma, q}\right), \quad \alpha:=\frac{1-\eta}{\eta(q-\sigma)},
$$


and according to [9], Theorem 12, this is the same as

$$
\Delta_{0, \varepsilon}^{(\infty)}\left(M(\sigma)\left(L^{p}(\Omega), L^{q}(\Omega)\right)_{1-\sigma, \infty}\right)=\left(L^{p}(\Omega), L^{q}(\Omega)\right)_{w, \infty}
$$

where $w(t)=t^{-1} b(t)$.

Application of Theorem 2.1 now gives

$$
\operatorname{dist}_{L_{b}^{q)}(\Omega)}\left(f, L^{\infty}(\Omega)\right) \approx \limsup _{t \rightarrow 0} w(t) K\left(t, f ; L^{p}(\Omega), L^{q}(\Omega)\right)
$$

if $b(t) \rightarrow 0$ as $t \rightarrow 0$ and $b$ is equivalent to a non-decreasing function.

To simplify this formula, we use the Holmstedt formula [3]

$$
K\left(t, f ; L^{p}(\Omega), L^{q}(\Omega)\right) \approx\left(\int_{0}^{t^{\alpha}} f^{*}(s)^{p} d s\right)^{1 / p}+t\left(\int_{t^{\alpha}}^{1} f^{*}(s)^{q} d s\right)^{1 / q}
$$

where $1 / \alpha=1 / p-1 / q$. Since $b$ is slowly varying on $(0,1)$, we see that

$$
\|f\|_{\left(L^{p}(\Omega), L^{q}(\Omega)\right)_{w, \infty}} \approx \sup _{0<t<1} b(t)\left(\int_{t^{\alpha}}^{1} f^{*}(s)^{q} d s\right)^{1 / q} .
$$

Indeed,

$$
\begin{aligned}
w(t)\left(\int_{0}^{t^{\alpha}} f^{*}(s)^{p} d s\right)^{1 / p} & \leq c \sup _{0<t<1} t^{1 / q} b(t) f^{*}(t) \\
& \leq c \sup _{0<t<1} b(t)\left(\int_{t^{\alpha}}^{2 t^{\alpha}} f^{*}(s)^{q} d s\right)^{1 / q}
\end{aligned}
$$

Analogously,

$$
\limsup _{t \rightarrow 0} w(t) K\left(t, f ; L^{p}(\Omega), L^{q}(\Omega)\right) \approx \limsup _{t \rightarrow 0} b(t)\left(\int_{t^{\alpha}}^{1} f^{*}(s)^{q} d s\right)^{1 / q} .
$$

These formulae suggest the following result. Let the quasi-norm on $L_{b}^{q)}(\Omega)$ be defined by

$$
\|f\|_{L_{b}^{q)}(\Omega)}:=\sup _{0<t<1} b(t)\left(\int_{t}^{1} f^{*}(s)^{q} d s\right)^{1 / q}
$$

Then we have the following result. 
Theorem 4.3. Let $b$ be defined by (4.8) and suppose that $M$ is tempered. If $b(t) \rightarrow 0$ as $t \rightarrow 0$, and also for some $N>1, t^{-N} b(t)$ is non-increasing, then for all $f \in L_{b}^{q)}(\Omega)$,

$$
\operatorname{dist}_{L_{b}^{q)}(\Omega)}\left(f, L^{\infty}(\Omega)\right)=\limsup _{t \rightarrow 0} b(t)\left(\int_{t}^{1} f^{*}(s)^{q} d s\right)^{1 / q}, \quad 0<q<\infty .
$$

Proof. First we claim that the distance is bounded from below by the expression

$$
d(f):=\limsup _{t \rightarrow 0} I(t, f)
$$

where

$$
I(t, f)=b(t)\left(\int_{t}^{1} f^{*}(s)^{q} d s\right)^{1 / q} .
$$

Since the expression in (4.20) is not a $p$-norm, we argue as in the proof of Theorem 3.2. Consider the case $q \geq 1$. (The proof when $0<q<1$ is similar.) For any small $\varepsilon>0$ and $g \in L^{\infty}(\Omega)$ we have

$$
I(t, f) \leq b(t)\left(\int_{t}^{1}(f-g)^{*}((1-\varepsilon) s)^{q} d s\right)^{1 / q}+b(t)\left(\int_{t}^{1} g^{*}(\varepsilon s)^{q} d s\right)^{1 / q} .
$$

Hence, using the monotonicity property of $b$, we obtain

$$
I(t, f) \leq(1-\varepsilon)^{-N-1 / q}\|f-g\|_{L_{b}^{q)}(\Omega)}+b(t)\|g\|_{L^{\infty}(\Omega)} .
$$

Taking the limit as $t \rightarrow 0$, we see that

$$
d(f) \leq(1-\varepsilon)^{-N-1 / q}\|f-g\|_{L_{b}^{q)}(\Omega)},
$$

which establishes our claim. For the reverse inequality we use the same approximating functions $f_{k}$ as in the proof of Theorem 3.1. From (3.10) and (3.11) we have

$$
\left\|f-f_{k}\right\|_{L_{b}^{q)}(\Omega)} \leq \sup _{0<t \leq \mu_{f}(k)} b(t)\left(\int_{t}^{1} f^{*}(s)^{q} d s\right)^{1 / q}
$$

and so

$$
\operatorname{dist}_{L_{b}^{q)}(\Omega)}\left(f, L^{\infty}(\Omega)\right) \leq \sup _{0<t \leq \mu_{f}(k)} b(t)\left(\int_{t}^{1} f^{*}(s)^{q} d s\right)^{1 / q} .
$$

All that remains is to take the limit as $k \rightarrow \infty$.

As a consequence we obtain the following result. 
Corollary 4.4. Let $0<q<\infty$ and define the quasi-norm on $L^{q)}(\Omega)$ by

$$
\|f\|_{\left.L^{q}\right)(\Omega)}=\sup _{0<t<1}(1-\log t)^{-1 / q}\left(\int_{t}^{1} f^{*}(s)^{q} d s\right)^{1 / q}
$$

Then for all $f \in L^{q)}(\Omega)$,

$$
\operatorname{dist}_{L^{q)}(\Omega)}\left(f, L^{\infty}(\Omega)\right)=\limsup _{t \rightarrow 0}(1-\log t)^{-1 / q}\left(\int_{t}^{1} f^{*}(s)^{q} d s\right)^{1 / q}
$$

Further developments of these ideas are clearly possible.

\section{References}

1. Bennett, C. H. and Rudnick, K., On Lorentz-Zygmund spaces, Dissertationes Math. (Rozprawy Mat.) 180 (1980), 1-72.

2. Bennett, C. H. and Sharpley, R., Interpolation of Operators, Pure and Applied Mathematics 129, Academic Press, Boston, MA, 1988.

3. Bergh, J. and Löfström, J., Interpolation Spaces, Grundlehren der Mathematischen Wissenschaften 223, Springer-Verlag, Berlin-New York, 1976.

4. Carozza, M. and Sbordone, C., The distance to $L^{\infty}$ in some function spaces and applications, Differential Integral Equations 10 (1997), 599-607.

5. EDMunds, D. E. and TRIEBEL, H., Function Spaces, Entropy Numbers, Differential Operators, Cambridge Tracts in Mathematics 120, Cambridge Univ. Press, Cambridge, 1996.

6. Garnett, J. B. and Jones, P. W., The distance in BMO to $L^{\infty}$, Ann. of Math. 108 (1978), 373-393.

7. IWANiec, T. and Sbordone, C., On the integrability of the Jacobian under minimal hypotheses, Arch. Rational Mech. Anal. 119 (1992), 129-143.

8. Jawerth, B. and Milman, M., Extrapolation theory with applications, Mem. Amer. Math. Soc. 89 (1991).

9. Karadzhov, G. E. and Mrlman, M., Extrapolation theory: new results and applications, Preprint 1, Inst. Math. and Informatics, Bulgarian Acad. Sciences, 2002.

10. Neves, J. S., Fractional Sobolev-type Spaces and Embeddings, Ph.D. Thesis, University of Sussex, Brighton, 2001. 
11. Triebel, H., Interpolation Theory, Function Spaces, Differential Operators, VEB Deutscher Verlag der Wissenschaften, Berlin, 1978; 2nd (revised) ed., Johann Ambrosius Barth, Heidelberg, 1995.

Received May 27, 2003

in revised form December 12, 2003
David E. Edmunds

Department of Mathematics

University of Sussex

Brighton BN1 9RF

United Kingdom

email: d.e.edmunds@sussex.ac.uk

Georgi E. Karadzhov

Institute of Mathematics and Informatics

Bulgarian Academy of Sciences

BG-1113 Sofia

Bulgaria

email: geremika@math.bas.bg 